\title{
MASS ENHANCEMENT AND CRITICAL BEHAVIOR IN TECHNICOLOR THEORIES
}

\author{
Thomas Appelquist, John Terning \\ Department of Physics, Yale University, New Haven, CT 06520 \\ and \\ L.C.R. Wijewardhana \\ Department of Physics, University of Cincinnati, Cincinnati, OH 45221
}

September 15, 2003

\begin{abstract}
Quark and lepton masses in technicolor theories can be enhanced if the high energy, extended technicolor (ETC) interactions play an important role in electroweak symmetry breaking. This happens when the ETC coupling and the technicolor gauge coupling at high energies lie close to a certain critical line. The enhancement has been associated with the existence of composite scalars made mainly of technifermions, with masses small compared to the ETC scale. The initial study of these states was carried out with the technicolor gauge coupling neglected. In this paper we investigate the properties of such scalars including the technicolor gauge interactions. We find that for realistic values of the gauge coupling, the scalars will not be narrow resonances. Mass and width estimates are made and some comments about the phenomenology of these states are included.
\end{abstract}

The recent revival of interest in technicolor theories of electroweak symmetry breaking has been stimulated partly by the observation that these theories should not necessarily be viewed as scaled-up versions of QCD. Momentum components well above the confinement scale $\Lambda_{t c}$ can play a more important role than they do in QCD - with important consequences such as the generation of very large fermion masses. Walking technicolor [1] is one example of this phenomenon. Another possibility is that the higher energy extended technicolor (ETC) interactions, which must be 
present to generate the masses of ordinary fermions, can play an important direct role, along with the technicolor interactions, in the electroweak breaking, leading to even larger fermion masses $[2,3]$. This can take place only if the combination of the ETC coupling and the technicolor coupling at the ETC scale is sufficiently close to a certain critical curve [4].

It has recently been suggested that this ETC-driven enhancement is associated with the appearance of composite scalars that are light compared to the ETC scale [5]. The enhanced fermion mass then arises from an effective Yukawa coupling of the fermion to the scalar which develops a vacuum value from the technicolor interactions. Here we summarize a study of the light composite scalars generated by near-critical high energy interactions. We conclude that unless the technicolor coupling at the ETC scale is unrealistically weak and the ETC coupling is very close to the critical curve, these light states will have large widths.

We consider a single doublet of technifermions $\Psi=(U, D)$ subject to a confining technicolor force and an additional, higher energy, attractive ETC interaction. The latter is approximated by an effective, $S U(2)_{L} \times U(1)$ invariant, four-fermion coupling [6]

$$
\mathcal{L}_{4 f}=\frac{8 \pi^{2} \lambda}{N_{t c} \Lambda^{2}}\left(\bar{\Psi}_{L}^{i} U_{R}\right)\left(\bar{U}_{R} \Psi_{L}^{i}\right)
$$

where $i$ is a summed $S U(2)_{L}$ index, $N_{t c}$ is the number of technicolors, $\Lambda$ is the ETC mass scale, and $\lambda$ is the interaction strength of the ETC interactions. Implicit technicolor indices are also summed in each fermion bilinear.

Only the $U_{R}$ and not the $D_{R}$ has been included in the ETC interaction, assuming that terms involving the latter will be weaker. Thus, while the technicolor 
interactions will generate a dynamical mass for both the $\mathrm{U}$ and D, the above ETC interaction will contribute only to the $\mathrm{U}$ mass. This anticipates that when ETC couplings to ordinary fermions are included, the mechanism being explored here will be especially important for the generation of the t-quark mass.

We begin by recalling some features of dynamical chiral symmetry breaking driven by the combination of a gauge interaction and a high energy, ETC, fourfermion interaction. Suppose that the physics of interest takes place at energies well above the confinement scale $\Lambda_{t c}$. It should be possible to describe this physics in terms of $\lambda$ and $\alpha(\Lambda)$ (the technicolor coupling at the ETC scale), with technicolor running neglected to first approximation. The running of the technicolor coupling can then be included perturbatively. We will adopt this procedure and return later to a discussion of the conditions under which the approach is reliable.

With running neglected, dynamical mass generation can be studied in linearized ladder approximation [4, 7]. In Landau gauge [8], the gap equation for the dynamical mass $\Sigma(p)$ of the $\mathrm{U}$ takes the following form after angular integration:

$$
\Sigma(p)=\frac{3 \alpha C_{2}(R)}{4 \pi} \int^{\Lambda^{2}} \frac{d k^{2}}{M^{2}} \Sigma(k)+\lambda \int^{\Lambda^{2}} \frac{d k^{2}}{\Lambda^{2}} \Sigma(k)+\ldots
$$

where $\alpha \equiv \alpha(\Lambda), C_{2}(R)$ is the quadratic Casimir of the technifermion representation $R, M$ is the maximum of $p$ or $k$, and the ellipses represent terms of order $\alpha^{2}, \alpha \lambda$, and higher.

Analysis of this equation in the $\alpha, \lambda$ plane [4] reveals that a critical curve separates the broken phase $(\Sigma \neq 0)$ from the symmetric phase $(\Sigma=0)$. For $\lambda \leq 1 / 4$, the broken phase exists only for $\alpha>\alpha_{c} \equiv \pi / 3 C_{2}(R)$. For $\lambda \geq 1 / 4$, the critical curve 
separating the two phases is defined by

$$
\lambda_{\alpha}=\left[\frac{1}{2}+\frac{1}{2} \eta\right]^{2}
$$

where $\eta \equiv \sqrt{1-\alpha / \alpha_{c}}$. The broken phase then exists only for $\lambda>\lambda_{\alpha}$.

When the running of the technicolor gauge coupling is re-introduced, this distinction between broken and symmetric phases is blurred. The growth of the coupling at momenta near $\Lambda_{t c}$ will in fact always break the chiral symmetry at these lower scales. The critical curve in $\lambda$ and $\alpha$ is therefore, loosely speaking, the dividing line between the regime where the high energy interactions (four-fermion and technicolor combined) are able to break the symmetry, and the regime where they are too weak. With $\lambda$ and $\alpha$ in the weak, "symmetric" regime the low energy breaking of the symmetry by the growth of the technicolor coupling near $\Lambda_{t c}$ will typically lead to $\Sigma(0) \sim \Lambda_{t c} \ll \Lambda$.

It is this regime, where the spontaneous breaking is dominated by the "low energy" technicolor interaction, that is of principal interest in this paper. To set the stage, however, we first suppose that $\alpha$ and $\lambda$ lie in the "broken" phase. The behavior of $\Sigma(p)$, as the critical curve is approached can be derived from the gap equation. Since in a realistic theory $\alpha<\alpha_{c}$, we summarize the critical behavior only for this case. As $\lambda \rightarrow \lambda_{\alpha}$ with $\alpha$ fixed, one finds that [4]

$$
\frac{\Sigma(0)}{\Lambda} \sim\left(\lambda / \lambda_{\alpha}-1\right)^{\frac{1}{2 \eta}}
$$

Thus the critical behavior involves a (gauge independent) [8] anomalous dimension that depends on the strength of the long range interaction. With technicolor running reinstated, this behavior will persist as long as $\lambda / \lambda_{\alpha}-1$ is such that $\Sigma(0) \gg \Lambda_{t c}$. 
We now return to the regime $\lambda<\lambda_{\alpha}$, where $\Sigma(0) \sim \Lambda_{t c}$. There it has been shown that for a range of $\lambda$ near $\lambda_{\alpha}$, the high energy mass of the technifermion $\Sigma(\Lambda)$ takes the form [3]

$$
\Sigma(\Lambda) \sim \frac{g^{2}<\bar{\psi} \psi>_{\lambda=0}}{\Lambda^{2}\left(1-\lambda / \lambda_{\alpha}\right)}
$$

Here, $<\bar{\psi} \psi>_{\lambda=0}$ is the technifermion condensate in a pure technicolor theory normalized at $\Lambda$, and $g^{2}=4 \pi^{2} \lambda$. If an ordinary fermion (quark or lepton) is coupled to the technifermion by an ETC interaction with strength of order $g^{2} / \Lambda^{2}$, then its mass is also given by Eq. 5. This expression exhibits the ETC-driven mass enhancement as $\lambda \rightarrow \lambda_{\alpha}$. It will break down once $\Lambda\left(1-\lambda / \lambda_{\alpha}\right)^{1 / 2} \sim \Lambda_{t c}$.

In Ref. [5], it was suggested that this enhancement can be attributed to the existence of a light scalar particle of mass $M \sim \Lambda\left(1-\lambda / \lambda_{\alpha}\right)^{1 / 2}$ [10]. It couples to the technifermion and then develops a vacuum value due to the technicolor interactions, producing a "tadpole" diagram and leading to the result (5). This estimate is reliable as long as $\lambda_{\alpha}-\lambda$ is large enough that $M \gg \Lambda_{t c}$. The discussion of scalar formation in Ref. [5] was restricted to a pure four-fermion theory [10], i.e. a NambuJona-Lasinio (NJL) model [11]. Here we include the technicolor gauge interactions and specifically address the issue of the existence of a light physical scalar.

To get an idea of what to expect, recall that in the regime outside the critical curve, the high energy interactions are strong enough to break the chiral symmetry and produce three massless scalar Goldstone bosons. They will also produce a massive scalar whose mass vanishes as the critical curve is approached. Since the phase transition in the non-running theory is second order [4], corresponding scalar resonances produced by the high energy interactions are also expected on the weak side 
of the critical curve, whose masses approach zero as the critical curve is approached. In this phase, still neglecting running, chiral symmetry ensures that the scalar resonances are degenerate. To explore the region of this phase near the critical curve, auxillary fields are introduced for these light scalar degrees of freedom.

Four auxiliary fields are introduced by replacing the effective four-fermion interaction in Eq. 1 by

$$
\mathcal{L}_{a u x}=-\frac{g}{\sqrt{N_{t c}}} \bar{\Psi}_{L} M\left(\begin{array}{c}
U_{R} \\
0
\end{array}\right)+\text { h.c. }-\frac{\Lambda^{2}}{4} \operatorname{Tr} M^{\dagger} M
$$

where $M=\sigma+i \vec{\tau} \cdot \vec{\pi}$. The $S U(2)_{L} \times U(1)$ symmetry is manifest in this Lagrangian. These fields do not, in general, have definite parity, since the original interaction (Eq. 1) is parity violating. We will refer to all of these fields as scalars. The study of critical behavior in terms of the auxiliary fields begins with the quadratic term in the effective potential. The classical piece can be read off from $\mathcal{L}_{\text {aux }}$ and the quantum piece can be computed in ladder approximation by evaluating the graphs depicted in Fig. 1 at zero external momentum $[12,8]$. The sum of graphs can be written as

$$
V_{\text {ladder }}^{(2)}=\frac{\left(\sigma^{2}+\vec{\pi}^{2}\right)}{2} g^{2} \int \frac{d^{4} k}{(2 \pi)^{4}} \frac{\Gamma(k)}{k^{2}}
$$

where $\Gamma(k)$ is the full ladder-approximation to the 1PI $\sigma U \bar{U}$ vertex with zero momentum flowing along the scalar line. $\Gamma(k)$ can be evaluated by solving the DysonSchwinger equation which gives [8]

$$
\Gamma(k)=\frac{1}{\frac{1}{2}+\frac{1}{2} \eta}\left(\frac{k^{2}}{\Lambda^{2}}\right)^{-\frac{1}{2}+\frac{1}{2} \eta} .
$$

Substituting this expression into Eq. 7 and combining the result with the classical 
term then gives the full quadratic effective potential

$$
V^{(2)}=\frac{\Lambda^{2}}{2}\left[1-\frac{\lambda}{\left(\frac{1}{2}+\frac{1}{2} \eta\right)^{2}}\right]\left(\sigma^{2}+\vec{\pi}^{2}\right) .
$$

The stability of this potential at the origin is determined by the sign of the coefficient of $\frac{1}{2}\left(\sigma^{2}+\vec{\pi}^{2}\right)$. The critical curve (Eq. 3 ) is determined by setting this coefficient to zero.

In the regime $\lambda<\lambda_{\alpha}$ of special interest here, the quadratic effective potential gives the zero-momentum limit of the scalar inverse propagator, that is the "zeromomentum mass"

$$
M(0)=\Lambda\left(1-\lambda / \lambda_{\alpha}\right)^{1 / 2}
$$

$M(0)$ will be small compared to $\Lambda$ if nature provides us with a $\lambda$ close to $\lambda_{\alpha}$. Recalling that the scalar $\sigma$ will develop a small vacuum value, we recover the fermion mass enhancement formula (Eq. 5) [3], together with its tadpole interpretation [5].

To explore the new physics at scales well below $\Lambda$ generated by near-critical high energy interactions, it is necessary to move beyond zero momentum. This amounts to the construction of the effective action of the low energy theory. The question of whether the light scalars exist as narrow or even broad resonances can be addressed by constructing the quadratic term in the effective action of the scalar fields. For the $\sigma$ field, for example, it can be written in the Euclidean-space form

$$
W^{(2)}[\sigma]=-\int \frac{d^{4} p}{(2 \pi)^{4}} \frac{1}{2} \Delta^{-1}(p) \sigma(p) \sigma(-p),
$$

where $\sigma(p)$ is the momentum-space field and $\Delta^{-1}(p)$ is the inverse scalar propagator at momentum $p$.

To compute $\Delta^{-1}(p)$, one must evaluate the graphs shown in Fig. 1 with some 
non-zero external momentum $p$ in the scalar legs [13]. This requires knowledge of $\Gamma(p, k)$, the full ladder-approximation to the $1 \mathrm{PI} \sigma U \bar{U}$ vertex with momentum $p$ flowing along the scalar line. A complete computation of $\Gamma(p, k)$ is difficult but an approximation will be adequate for our purposes. We begin by considering a Taylor series expansion about $p=0$ of $\Delta^{-1}(p)$. In the ladder approximation one can show, by use of the Dyson-Schwinger equation for $\Gamma(p, k)$, that two terms contribute to the second derivative (with respect to $p^{\mu}$ ) of the graphs in question (See Fig 2). One term has two derivatives on a fermion propagator and a $\Gamma(p, k)$ at each scalar vertex; the other term has one derivative on a propagator, a $\Gamma(p, k)$ at one vertex, and a derivative of $\Gamma(p, k)$ at the other vertex. The derivative of $\Gamma(p, k)$ is higher order in $\alpha$ than $\Gamma(p, k)$. By solving the Dyson-Schwinger equation for the partial derivative of $\Gamma(p, k)$ with respect to $p$, evaluated at $p=0$, one can show that the second term is also numerically smaller than the first (evaluated at $p=0[14]$ ) for $\alpha \leq \alpha_{c}$.

Higher derivatives of $\Delta^{-1}(p)$ will also contain terms with and without derivatives of $\Gamma(p, k)$. In what follows we will drop all terms involving derivatives of $\Gamma(p, k)$, assuming their sum is numerically smaller than the sum of terms with no derivatives of $\Gamma(p, k)$. These terms in the Taylor expansion contain only graphs with derivatives on the fermion propagator. They can easily be summed.

This resummation gives a graph with a $\Gamma(k)$ at each scalar vertex, and the external momentum $p$ flowing through one fermion line. Performing the trace and the angular integrations we obtain

$$
\Delta^{-1}(p)-\Delta^{-1}(0)=\frac{g^{2}}{8 \pi^{2}} \int_{0}^{p^{2}} d k^{2} \Gamma^{2}(k)\left(\frac{k^{2}}{p^{2}}-2\right)-p^{2} \frac{g^{2}}{8 \pi^{2}} \int_{p^{2}}^{\Lambda^{2}} d k^{2} \frac{\Gamma^{2}(k)}{k^{2}} .
$$


Combining the result for the effective potential (Eq. 9) with the above kinetic term (and Wick rotating so that $p^{2}$ is positive for a time-like Minkowski momentum) we find

$$
\Delta^{-1}(p)=-\Lambda^{2}\left(a \frac{p^{2}}{\Lambda^{2}}+b\left(\frac{p^{2}}{\Lambda^{2}}\right)^{\eta}[\cos (\eta \pi)-i \sin (\eta \pi)]+1-\frac{\lambda}{\lambda_{\alpha}}\right)
$$

where

$$
\begin{aligned}
& a=\frac{\lambda}{2 \lambda_{\alpha}(1-\eta)}, \\
& b=\frac{\lambda}{\lambda_{\alpha} \eta\left(1-\eta^{2}\right)} .
\end{aligned}
$$

Recall that we are restricting our attention to the case $\lambda>1 / 4\left(\alpha<\alpha_{c}\right)$. Before proceeding further with the question of the possible existence of narrow resonances for realistic values of the coupling constants, we examine Eq. 13 in some simple limiting cases.

In the NJL limit $(\alpha \rightarrow 0)$ we find

$$
\Delta_{N J L}^{-1}(p)=p^{2} \frac{\lambda}{2}\left[\ln \left(\frac{\Lambda^{2}}{p^{2}}\right)+i \pi\right]-\Lambda^{2}(1-\lambda) .
$$

Poles of $\Delta_{N J L}(p)$ occur for complex $p^{2}$. We parameterize the location of the pole nearest the physical region by a mass and a width, i.e., the pole occurs at $p^{2} \equiv$ $\left(M_{N J L}-\frac{i}{2} \Gamma_{N J L}\right)^{2}$. For $\lambda$ close to 1 this yields (keeping only leading terms in $\left.\Gamma_{N J L} / M_{N J L}\right)$ the physical mass

$$
M_{N J L}^{2} \approx \frac{2(1-\lambda)}{\lambda \ln \left(\frac{\lambda}{2(1-\lambda)}\right)} \Lambda^{2}
$$

and narrow width

$$
\frac{\Gamma_{N J L}}{M_{N J L}} \approx \frac{\pi}{\ln \left(\frac{\lambda}{2(1-\lambda)}\right)}
$$


Thus the familiar NJL results are recovered in this limit. To obtain Eq. 16 from Eq. 13, an expansion in $\frac{\alpha}{2 \alpha_{c}} \ln \left(\frac{\Lambda^{2}}{p^{2}}\right)$ and $\frac{\alpha}{2 \alpha_{c}}$ has been made, keeping only the zeroth order term. Note that this means dropping a term of order $\lambda \frac{\alpha}{2 \alpha_{c}}$ relative to the $1-\lambda$ term. To obtain Eqs. 17 and 18 we have assumed that $\ln \left(\frac{\lambda}{2(1-\lambda)}\right) \gg 1$. Therefore these two equations should give good approximations for the mass and width only when

$$
\frac{\alpha}{2 \alpha_{c}} \ll \frac{1-\lambda}{\lambda} \ll \frac{1}{\ln \left(\frac{\lambda}{2(1-\lambda)}\right)} \ll 1 .
$$

For the more realistic case of finite $\alpha$, we examine the location of the poles of the propagator as $\lambda$ approaches $\lambda_{\alpha}$. The poles again occur for complex $p^{2}$ so we set $p^{2}=p_{0}^{2} \exp (-i \theta)$. For $\eta<1$, and $\lambda$ very close to $\lambda_{\alpha}$, we can neglect $\frac{p^{2}}{\Lambda^{2}}$ relative to $\left(\frac{p^{2}}{\Lambda^{2}}\right)^{\eta}$ in the real part of $\Delta^{-1}(p)$. We then find zeros of $\Delta^{-1}(p)$ at

$$
\begin{gathered}
p_{0} \approx \Lambda\left(\frac{1-\frac{\lambda}{\lambda_{\alpha}}}{b}\right)^{\frac{1}{2 \eta}}, \\
\theta \approx\left(\frac{m-\eta}{\eta}\right) \pi+\frac{a \sin \left(\frac{m-\eta}{\eta} \pi\right)}{b \eta}\left(\frac{1-\frac{\lambda}{\lambda_{\alpha}}}{b}\right)^{\frac{1-\eta}{\eta}},
\end{gathered}
$$

where $m$ is an odd integer. We expect the physical pole to correspond to $m=1$, since it is the closest pole to the physical region. Eq. 20 describes how the mass scale of the pole position vanishes as $\lambda$ is tuned toward the critical curve. It is interesting to note that this scaling law is different from that of the zero-momentum mass of the scalar (Eq. 10). It is, however, the same scaling law as exhibited by $\Sigma(0)$ in the broken phase (Eq. 4). We expect the same scaling behavior for the mass of the scalar in the broken phase. Eqs. 20 and 21 give a reliable description of the pole position of $\Delta(p)$ only when

$$
a\left(\frac{1-\frac{\lambda}{\lambda_{\alpha}}}{b}\right)^{\frac{1}{\eta}} \ll 1-\frac{\lambda}{\lambda_{\alpha}} .
$$


It can be seen from this condition that the scaling behavior sets in more rapidly as $\alpha$ is increased toward $\alpha_{c}$.

We next consider under what conditions the scalar resonance will be narrow. With a pole located at $p^{2}=p_{0}^{2} \exp (-i \theta), \Delta(p)$ will describe a narrow resonance if $\theta$ is small. In that case, it is reasonable to parameterize the location of the pole by a mass and a width. That is, we set $p_{0}^{2} \exp (-i \theta)=\left(M_{\sigma}-\frac{i}{2} \Gamma_{\sigma}\right)^{2}$, which yields

$$
\begin{gathered}
M_{\sigma}=p_{0}\left(\frac{1+\cos (\theta)}{2}\right)^{\frac{1}{2}} \approx p_{0}, \\
\frac{\Gamma_{\sigma}}{M_{\sigma}}=\frac{2 \sin (\theta)}{1+\cos (\theta)} \approx \theta .
\end{gathered}
$$

When is $\theta$ in fact small? We first observe that for finite $\alpha(\eta<1), \theta$ (Eq. 21) does not approach zero as $\lambda \rightarrow \lambda_{\alpha}$. Therefore, the width to mass ratio is not suppressed (as in the NJL case, $\alpha=0$ ) as the critical curve is approached. For small but nonzero $\alpha$, this expression gives $\theta \rightarrow \frac{\alpha}{2 \alpha_{c}} \pi$ as $\lambda \rightarrow \lambda_{\alpha}$. Thus, as the mass scale of the scalar state is made small by approaching the critical curve, it is not described by a narrow Breit-Wigner resonance unless $\alpha$ is quite small.

Having considered these special limiting cases, we now consider more generic values of the coupling constants. A description of the resonance structure of the theory is provided by a plot of $\operatorname{Im} \Delta(p)$. We do this by evaluating the general expression for $\Delta(p)$ Eq. 13, and plotting $\operatorname{Im} \Delta(p)$ versus $p / M(0)$ (in Fig. 3) for various values of $\lambda / \lambda_{\alpha}$ and $\alpha / \alpha_{c}$. Each figure corresponds to a different (small) value of $M(0) / \Lambda=\left(1-\lambda / \lambda_{\alpha}\right)^{1 / 2}$. In each case, a resonant curve exists for the smallest value of $\alpha / \alpha_{c}$, peaked at a momentum smaller than $M(0)$. The curve then shifts down (relative to $M(0))$ and broadens (relative to the position of the peak) as $\alpha / \alpha_{c}$ is increased. Both the shifting down of the peak and the broadening are 
consistent with the limiting cases discussed above.

As a specific example, consider the case in which $\lambda$ is tuned to within $1 \%$ of $\lambda_{\alpha}$ (Fig. 3b), giving $M(0) / \Lambda \approx 1 / 10$. The likely value of $\alpha(\equiv \alpha(\Lambda))$ depends on the details of the technicolor theory. If $\Lambda$ is in a range between, say, $30 \mathrm{TeV}$ and $1000 \mathrm{TeV}$, and if the technicolor coupling either runs normally or walks at a rate attainable in a realistic theory, $\alpha / \alpha_{c}$ will be somewhere between roughly 0.2 and 0.5. This is a range within which a broad Breit-Wigner curve exists, peaked roughly around $0.3 M(0)$. With $\Lambda=100 \mathrm{TeV}$, for example, the peak would be around $3 \mathrm{TeV}$, with a full width at half maximum of roughly the same order. With less fine tuning (Fig. 3a), the resonance is broad even in the NJL limit. With a great deal of fine tuning (Fig. 3d), the resonance is narrow for very small $\alpha$ but becomes broad once $\alpha / \alpha_{c}$ is as large as say 0.2 .

The curves of Fig. 3 show clearly that the tightly bound light scalar objects produced by near-critical high energy interactions will not in general be narrow resonances. Even with $\lambda$ tuned very close to $\lambda_{\alpha}$, the width to mass ratio will only be small if $\alpha$ is unrealistically small for a technicolor theory.

To understand the origin of these results, it is convenient to frame the discussion in terms of the wavefunction renormalization factor $Z$ of the scalar. In the NJL limit, $Z$ is sensitive to high momentum components and is proportional to $\ln \Lambda^{2} / p^{2}$. The scalar couplings to fermions are inversely proportional to $Z^{1 / 2}$. Therefore, these states are weakly coupled in the limit $\Lambda^{2} / p^{2} \gg 1$, which arises when $\lambda-1 \ll 1$. It is not surprising then that the NJL resonances can be narrow (Eq. 18). The effect of the long range gauge interaction is to shift the sensitivity of $Z$ towards the infrared. $Z^{1 / 2}$ will be large (proportional to $\left.\left(\Lambda^{2} / p^{2}\right)^{1 / 2-\eta / 2}\right)$ due to 
the contribution of small momentum components. This large denominator factor, however, will be cancelled by a corresponding $\Lambda$ dependence in $\Gamma(k)$ (Eq. 8), which also enters into the computation of the decay widths. The partial and full decay widths depend only on momentum components at the scale of the resonance peak and are independent of $\Lambda$. There is therefore no reason for the resonances to be narrow.

In a more realistic theory, there will also be strong ETC interactions coupling the $\mathrm{t}$ quark to the $\mathrm{U}$, as well as to itself. The $\mathrm{t}$ is therefore expected to play a role equal to the $\mathrm{U}$ in the determination of critical behavior and the enhancement of fermion mass. Even though its coupling to the scalar channels will be strong at the ETC scale, it will be much more weakly coupled than the $\mathrm{U}$ at the scale associated with the resonances. This can be seen directly from the above discussion. The Z factor of the scalar is large (as described above) in the region of the resonance. We are assuming here that only one set of parameters is tuned close to critical and that there is therefore only one set of light scalars. Since the $t$ has no technicolor interactions, however, there is no vertex correction to compensate this factor.

For any of our conclusions to be useful in a technicolor theory, it is important to discuss the effect on the light physics of reinstating the running of the technicolor coupling. We have already pointed out that the running turns what we have been calling the "symmetric" phase into a broken phase with the breaking taking place on the order of the confinement scale $\Lambda_{t c}$. This produces the fermion mass enhancement formula of Eq. 5 with its tadpole interpretation.

What effect does the running have on the resonance spectrum just discussed and other features of the low energy physics? First of all, the technifermions and 
the techni-gauge bosons will be confined at scales on the order of $1 / \Lambda_{t c}$. If the light scales discussed above (those appearing in the graphs of Fig. 3) are large compared to $\Lambda_{t c}$, the confinement can be expected to have little effect on the mass and total width. The ladder computations for the total width reported above will remain reliable in analogy to the way $\mathrm{QCD}$ can be used to describe $\mathrm{R}$ in $e^{+} e^{-}$annihilation. The dominant decay products of the scalar resonances will be the technicolor-singlet technihadrons, including the longitudinally polarized $W$ 's and $Z$ 's. The various partial decay rates will depend in detail on the confinement dynamics as they do in QCD.

If these resonances are not too far above $\Lambda_{t c}$, they may be accessible at the the SSC or at a very high energy $e^{+} e^{-}$collider. Since their strongest couplings are likely to be to the U-type techniquark, the dominant production mechanism will probably involve $U \bar{U}$ production followed by emission of the resonant state from one of these heavy fermions. Whether the resonances can be detected will depend on the partial and full widths, as well as detector capabilities, backgrounds, etc.

Whether the resonances lie enough above $\Lambda_{t c}$ so that our estimates neglecting the running are reliable, depends on $\alpha / \alpha_{c}$ and the smallness of $\lambda-\lambda_{\alpha}$. Suppose first that $\alpha / \alpha_{c} \equiv \alpha(\Lambda) / \alpha_{c}$ is small, say, $\approx 0.1$. If $\Lambda$ is in the range from $100 \mathrm{TeV}$ to $1000 \mathrm{TeV}$, this corresponds to a normally running theory. If $\lambda-\lambda_{\alpha}$ is not too small, the resonance mass will then sit well above $\Lambda_{t c}$, where the running coupling remains quite close to $\alpha(\Lambda)$. In this case, it can also be seen that the small $\alpha$ expansion of $\Delta^{-1}(p)$ should be reasonably convergent. The use of the zeroth-order term (Eq. 16) should give a good first approximation. Higher order corrections involving both ladder exchange and running coupling corrections could then be 
computed simultaneously.

If $\alpha / \alpha_{c}$ is larger, then the anomalous dimension in $\Gamma(k)$ is large and the full form of $\Delta^{-1}(p)$ must be retained. Still, the neglect of running can be a good first approximation. Suppose that $\alpha / \alpha_{c} \approx 0.5$. With $\Lambda$ in the range between $100 \mathrm{TeV}$ and $1000 \mathrm{TeV}$, this corresponds to a rather slowly running theory. With $\lambda-\lambda_{\alpha}$ small but not too small, the resonance mass will be small compared to $\Lambda$ but well above $\Lambda_{t c}$. In this case, it is walking that justifies the use of a constant technicolor coupling as a first approximation. Computations showing the the effect of running in different cases will be reported in a future publication.

If the parameters are such that the resonance curves are centered at energies of order $\Lambda_{t c}$, then the computation of $\Delta(p)$ described above will not be quantitatively reliable. The zero-momentum mass $M(0)$ may still be above $\Lambda_{t c}$ and the estimate of Eq. 10 still reliable. Confinement effects, however, could become important in the description of the resonances which then could mix with the technicolor states. Disentangling the experimental signals in this case may be difficult.

An important question is whether the light scalar resonances are able to mediate flavor-changing neutral currents. If we restrict our attention to $\mathrm{CP}$ conserving interactions, then possible off-diagonal couplings of ordinary fermions to these resonances will not produce unacceptable flavor-changing neutral currents if the zero-momentum boson masses, $M(0)$, are above $1.5 \mathrm{TeV}[15]$. The contribution of the scalars to flavour-changing neutral current processes involving the t quark may be much larger. This could be of immediate interest if the $t$ is discovered in the next few years. 
Finally, it is worth pointing out that the results described here will not be qualitatively changed if the effective four-fermion interaction is replaced by a realistic interaction such as the exchange of a heavy spin-one boson [6]. The low energy physics doesn't depend on the details of the high energy physics, but only on the symmetries and whether the couplings are tuned relatively close to criticality.

To conclude, we have studied the properties of light composite scalars which are present in technicolor theories with near-critical ETC interactions. We have shown that these scalars (which can enhance quark and lepton masses) will not be narrow resonances for realistic values of the technicolor gauge coupling. In addition we have pointed out some phenomenological consequences of these resonances.

\section{Acknowledgements}

We thank S. Chivukula, A. Cohen, D. Kosower, K. Lane, S. Macdowell, and V. Miransky for helpful discussions. This work was supported in part by the Natural Sciences and Engineering Research Council of Canada and by the U.S. Department of Energy under contracts DE-AC-02-76ERO3075 and DE-FG-02-84ER40153. T.W.A and L.C.R.W. acknowledge the Aspen Center for Physics for its hospitality during the summer of 1990 . 


\section{Figure Captions}

Fig. 1. The quantum corrections to the quadratic effective action. The wavy lines are techni-gauge bosons, the solid lines are technifermions, and the dashed lines represent the scalar field.

Fig. 2. The second derivative of $\Delta^{-1}(p)$. The external momentum $p$ flows through the upper lines, and the slashes indicate a derivative with respect to $p_{\mu}$.

Fig. 3. Graphs of $\operatorname{Im} \Delta(p)$ for different values of $\lambda / \lambda_{\alpha}$, and different values of $\alpha / \alpha_{c}$. The curves in each graph are normalized so that the peak value of the $\alpha / \alpha_{c}=0.01$ curve equals 1 . 


\section{References}

[1] B.Holdom, Phys. Lett. B150, 301 (1985); T. Appelquist, D. Karabali, and L.C.R. Wijewardhana, Phys. Rev. Lett. 57, 957 (86); T. Appelquist and L.C.R. Wijewardhana, Phys. Rev. D35, 774 (87), and Phys. Rev. D36, 568 (1987).

[2] T. Appelquist, M. Einhorn, T. Takeuchi, and L.C.R. Wijewardhana, Phys. Lett. 220B, 1043 (1989).

[3] T. Takeuchi, Phys. Rev. D40, 2697 (1989).

[4] T. Appelquist, M. Soldate, T. Takeuchi, and L.C.R. Wijewardhana in "Proceedings of the 12th Johns Hopkins Workshop on Current Problems in Particle Theory, ed. by G. Domokos and S. Kovesi-Domokos, World Scientific (1988); K.I. Kondo, H. Mino and K. Yamawaki, Phys. Rev. D39, 2430 (1989); M. Inoue, T. Muta, and T. Ochiumi, Mod. Phys. Lett. A4, 605 (1989); K.I. Aoki, Kyoto preprint, RIFP-758 (1988).

[5] R.S. Chivukula, A. Cohen, and K. Lane, Nucl. Phys. B343, 554 (1990).

[6] For a discussion of ETC-driven enhancement with massive ETC boson exchange replacing the four-fermion interaction, see T. Appelquist and O. Shapira, Phys. Lett. B249, 83 (1990); See also S. King and D. Ross, Southhampton University preprint, 1990.

[7] T. Appelquist, K. Lane, and U. Mahanta, Phys. Rev. Lett. 61, 1553 (1988); U. Mahanta, Phys. Rev. Lett. 62, 2349 (1989). 
[8] T. Appelquist, U. Mahanta, D. Nash, and L.C.R. Wijewardhana, Phys. Rev. D43, R646 (1991). The exponent in Eq. 8 is sometimes denoted as $\gamma_{m}$, the anomalous dimension of the fermion mass operator.

[9] T. Appelquist, D. Carrier, L.C.R. Wijewardhana, and W. Zheng, Phys. Rev. Lett. 60, 1114 (1987); R. Casalbuoni, S. DeCurtis, and R. Gatto, Nucl. Phys. B319, 367 (1989); S. King and D. Ross, Phys. Lett. B228, 363 (1989).

[10] In Ref. [5], the variation (Eq. 3) of $\lambda_{\alpha}$ with $\alpha$ was neglected. The authors simply took $\lambda_{\alpha}=1$.

[11] Y. Nambu and G. Jona-Lasinio, Phys. Rev. 122, 345 (1961).

[12] W. Bardeen, S.T. Love, and V. Miransky, Phys. Rev. D41, 3514 (1990).

[13] Because this computation includes the low momentum components of the massless fermions and gauge bosons, it is not a computation of the effective action in the usual low energy sense.

[14] When truncating at a finite number of terms in the Taylor series an infrared cutoff is required to make the result finite. No such cutoff is needed after the resummation is performed.

[15] S. Dimopoulos and J. Ellis, Nucl. Phys. 182 (1981) 505. 